\title{
Nuclear Isomers: Recipes from the Past and Ingredients for the Future
}

\author{
Philip M. Walker ${ }^{1}$ and James J. Carroll ${ }^{2}$ \\ ${ }^{1}$ Department of Physics, University of Surrey, Guildford, Surrey GU2 7XH, UK \\ ${ }^{2}$ Department of Physics and Astronomy, Youngstown State University, \\ Youngstown, Ohio 44555, USA
}

\section{Introduction}

“But you've no idea what a difference it makes, mixing it with other things" [1].

In this new age of radioactive beams for nuclear physics research, it is timely to reflect on the enduring role of nuclear isomers, i.e. excited, metastable states of nuclei. Heroic experiments with tiny quantities of isomer targets point the way towards the wide vistas of isomer beams, and new possibilities confront our ingenuity at the interface between nuclear and atomic physics. It is the relatively long half-lives of isomers that lead to their special status.

The prediction by Soddy in 1917 [2] that a single nuclide might have states which are "different in their stability and mode of breaking up" was soon followed by Hahn's observations in 1921 of $\mathrm{UZ}$ and $\mathrm{UX}_{2}$, now known as ${ }^{234} \mathrm{~Pa}$ and ${ }^{234 \mathrm{~m}} \mathrm{~Pa}$. However, isomers only became well recognised in 1936, with von Weizsäcker's explanation in terms of angular momentum: high angular momentum transitions are slow. So successful were isomers at accounting for multiple half-lives that, it can be argued, the discovery of uranium fission in 1938 had been significantly delayed. Isomers went on to be cornerstones of the nuclear shell model, and even the collective model owes much to isomers. A classic example is ${ }^{180 \mathrm{~m}} \mathrm{Hf}$, which decays through a sequence of states, interpreted by Bohr and Mottelson [3] as having energies characteristic of a quantum rotor. The energy gap associated with isomers in even-even nuclides also tells us much about the pairing interaction.

The place of isomers in contemporary nuclear structure investigations is illustrated in the following paragraphs. Other recent reviews include those of Walker and Dracoulis [4,5] and Walker and Carroll [6].

\section{Extreme isomers}

Most isomers can be characterised as shell-model states involving one or two - but even up to about ten - specific, unpaired nucleon orbitals. The isomer-related orbitals typically couple to make higher-spin states than other couplings at similar energy, so that isomer decay necessitates the emission of high-spin and/or low-energy radiation, resulting in their long half-lives. In the case of deformed nuclei with an axis of symmetry, a key feature is the orientation of the angular-momentum vector, with a projection, $\mathrm{K}$, on the symmetry axis that is approximately conserved, leading to the occurrence of $\mathrm{K}$ isomers. The close connection with shell-model orbits means that an isomer can be considered to be a "simple" state, with the bulk of the wavefunction being well defined. The blocking of pairing correlations in multi-particle isomers leads to them being, in a sense, even simpler than ground states. Nevertheless, the observed radiations from isomers frequently violate selection rules, and there is a 
sensitive dependence on low-amplitude wavefunction admixtures [7]. Some of these features are schematically illustrated in Figure 1. The remaining principal class of isomers is that of shape isomers, exemplified by fission isomers, where it is a shape change rather than an angular-momentum change that is responsible for the long halflife. Using an energy-spin representation, as shown in Figure 2, the different isomer types can be broadly separated by their general origins, although there are overlaps, such as $\mathrm{K}$ traps being also yrast traps if they lie on the yrast line (the locus of states with lowest energy at each spin value).

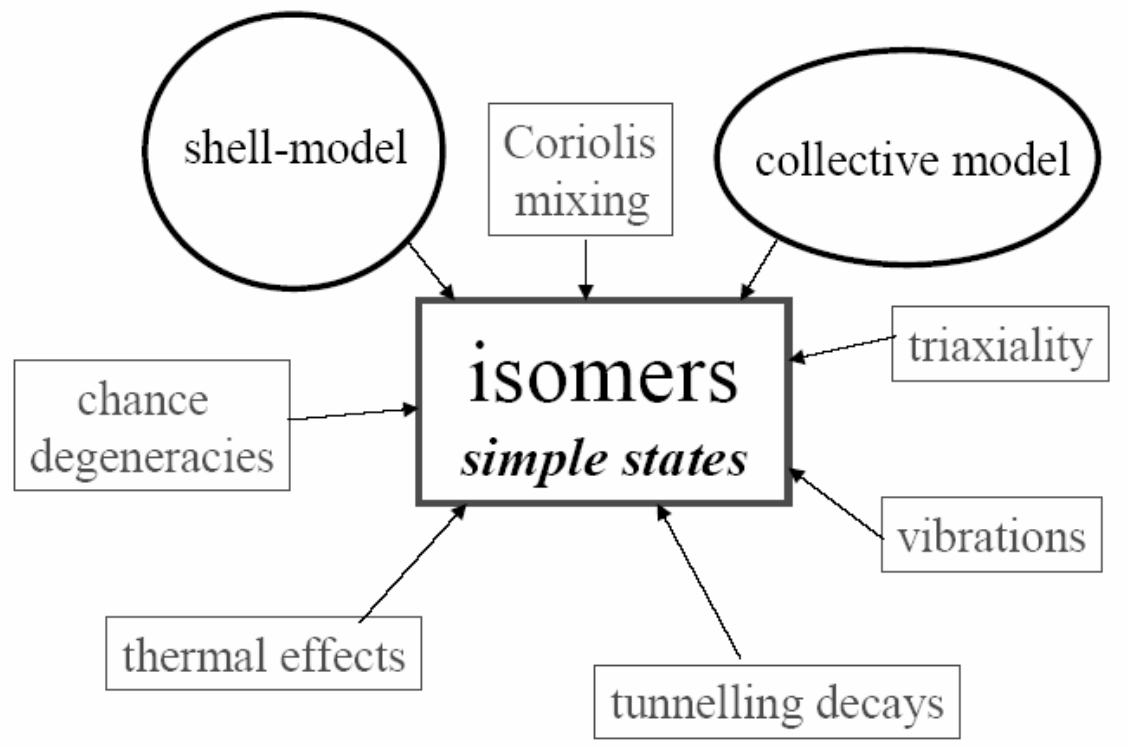

Figure 1: While isomers can be considered to have simple shell-model configurations, their decay radiations are sensitive to many influences.

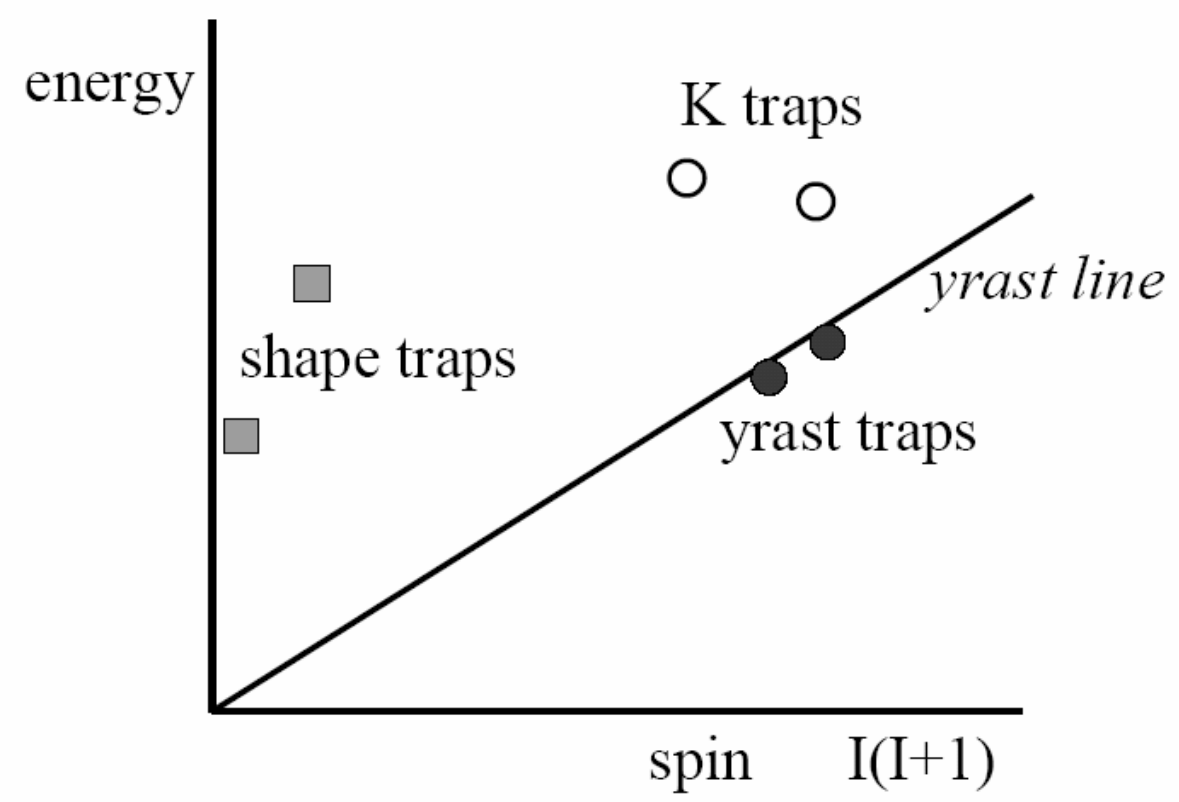

Figure 2: Schematic representation of isomer types in energy-spin space. 
In order to appreciate the range of isomers found in nature, we will discuss some extreme examples - see Table I. First, however, a comment is needed as to what halflife is necessary for an excited nuclear state to be called an "isomer". This half-life is ill-defined, but is usually taken to be long enough to permit an isomer and its decay radiations to be separated from the plethora of "prompt" radiations from nuclear reactions. For the purposes of the present discussion, the minimum half-life is taken to be $5 \mathrm{~ns}$.

\section{Table I: Examples of extreme isomers}

$\begin{array}{lcrlll}\text { Nuclide } & \text { Half-life } & \text { Spin (ћ) } & \text { Energy } & \text { Attribute } & \text { Ref } \\ { }^{12} \mathrm{Be} & \sim 500 \mathrm{~ns} & 0 & 2.2 \mathrm{MeV} & \text { low mass } & {[8]} \\ { }^{94} \mathrm{Ag} & 300 \mathrm{~ms} & 21 & 6 \mathrm{MeV} & \text { proton decay } & {[9]} \\ { }^{152} \mathrm{Er} & 11 \mathrm{~ns} & \sim 36 & 13 \mathrm{MeV} & \text { high spin and energy } & {[10]} \\ { }^{180} \mathrm{Ta} & >10^{16} \mathrm{y} & 9 & 75 \mathrm{keV} & \text { long half-life } & {[11]} \\ { }^{229} \mathrm{Th} & \sim 5 \mathrm{~h} & 3 / 2 & \sim 7.6 \mathrm{eV} & \text { low energy } & {[12]} \\ { }^{270} \mathrm{Ds} & \sim 6 \mathrm{~ms} & \sim 10 & \sim 1 \mathrm{MeV} & \text { high mass } & {[13]}\end{array}$

The half-life range in Table I covers 33 orders-of-magnitude. The observed decay modes, even from this short list, include $\alpha\left({ }^{270} \mathrm{Ds}\right), \beta\left({ }^{94} \mathrm{Ag}\right), \gamma\left({ }^{152} \mathrm{Er}\right), \mathrm{p}\left({ }^{94} \mathrm{Ag}\right)$ and $2 \mathrm{p}$ $\left({ }^{94} \mathrm{Ag}\right)$ emission. Note the absence of neutron emission. The ${ }^{152} \mathrm{Er}$ isomer at $13 \mathrm{MeV}$ is neutron (and proton) unbound, but such decay is inhibited by the centrifugal barrier. We can speculate that neutron radioactivity may soon be discovered from as-yetunknown long-lived isomers in neutron-rich nuclides.

The extremely low energy, $\sim 7.6 \mathrm{eV}$, of the ${ }^{229} \mathrm{Th}$ isomer has attracted considerable attention. One possible application is to test the constancy of fundamental constants [14].

An isomer can be longer lived than its ground state. For example, there is a factor of about sixty between the $\sim 6 \mathrm{~ms}$ isomer in ${ }^{270} \mathrm{Ds}$ and its $\sim 100 \mu$ s ground state. Indeed, this feature may apply more widely to superheavy nuclides [15] and thus could provide significant experimental advantages. Conversely, at the predicted superheavy "island of stability", if ground-state half-lives are too long (e.g. 1 hour) for the detection of time-correlated single-atom decays, it may yet be possible to identify decays from short-lived isomers. Perhaps there are already cases where superheavy isomers, rather than their ground states, have unknowingly been detected.

From this brief discussion, it is evident that, aside from interest in the structure of isomers themselves, they can serve as "tools" or "stepping stones" as we reach out to search for exotic phenomena, especially as we explore the limits of nuclear stability. Another example is ${ }^{254} \mathrm{No}$ [16] where highly excited isomers provide information about orbitals that may form the ground-state structure of heavier nuclides; and a wealth of new information is coming from projectile-fragmentation reactions, as performed at GANIL, GSI and NSCL. These reactions produce a vast range of nuclides, both proton rich and neutron rich, which can be identified by their mass-tocharge (A/q) ratio on an ion-by-ion basis, within about $500 \mathrm{~ns}$ of their formation. The best sensitivity to nuclear structure, through measuring the subsequent decay 
radiations, is for isomers that decay on a $\mu$ s time scale, which minimises random events. Early work of this kind was already sensitive to isomeric-ion rates of less than one per second, and experimental techniques are improving rapidly [17].

\section{Isomer targets}

In principle, isomers can be used for the full range of nuclear reactions that are open to ground states. However, there is only one naturally occurring, effectively "stable" isomer, ${ }^{180 \mathrm{~m}} \mathrm{Ta}$, and this is nature's rarest stable nuclide. On the positive side for isomer experiments, the ground state is unstable and $\beta$ decays with an 8-hour half-life, but natural tantalum is only $0.01 \%{ }^{180 \mathrm{~m}} \mathrm{Ta}$, the remainder being ${ }^{181} \mathrm{Ta}$. Despite the difficulties, enriched targets have been made, even in sufficient quantities for photon scattering experiments. Since the stellar nucleosynthesis and survival of ${ }^{180 \mathrm{~m}} \mathrm{Ta}$ present a long-standing puzzle, the laboratory photo-destruction of the isomer, through its ground state, gives vital information regarding possible stellar synthesis environments. Belic et al. [18] identified single-step photo-destruction resonances at photon energies down to $1010 \mathrm{keV}$. This was subsequently shown to fit well with level-scheme information, enabling the details of the photo-destruction pathway to be identified [19], as illustrated in Figure 3. Note that the minimum photon energy of $1010 \mathrm{keV}$ is, in this case, much higher than the isomer energy of $75 \mathrm{keV}$. In addition, multi-step photo-destruction at lower photon energies has been predicted to be important in stellar environments [20].

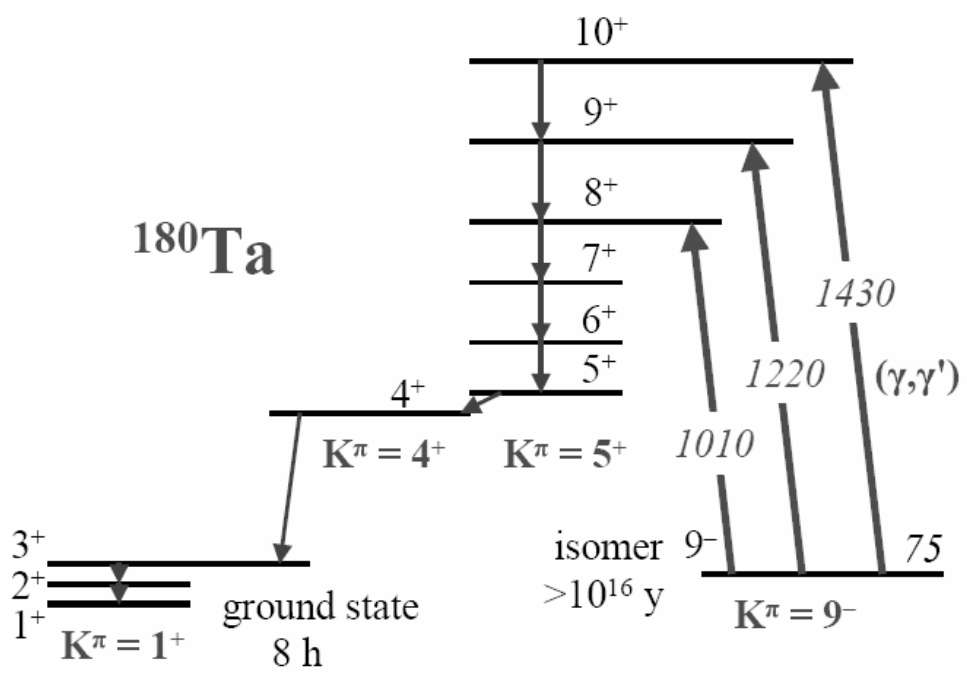

Figure 3: Simplified scheme showing ${ }^{180 m}$ Ta photo-excitation, with decay to the ground state [19]. Energies (in italics) are in keV.

Radioactive isomer targets have also been made. A remarkable case is ${ }^{178 \mathrm{~m} 2} \mathrm{Hf}$, a 31 year isomer at an excitation energy of $2.45 \mathrm{MeV}$. This has a unique combination of high energy and long half-life, arising from a $\mathrm{K}=16$, 4-quasiparticle configuration. Targets with more than $10^{14}$ atoms of ${ }^{178 \mathrm{~m} 2} \mathrm{Hf}$ have been used for several nuclear physics experiments, such as deuteron scattering [21], but target impurities have limited the quality of the data obtained. Attempts at photo-destruction of the isomer have been controversial [22], but so far only upper limits have been reliably determined [23] in the photon energy range $5-100 \mathrm{keV}$. 


\section{Isomer beams}

The question naturally arises, in view of the high intensities of radioactive beams soon to be available, as to whether isomers would make better beams than targets. Compare the background of $10^{5}$ decay/s from $10^{14}$ atoms of ${ }^{178 \mathrm{~m} 2} \mathrm{Hf}$, making up some small fraction of a target, with the near-zero background that could be obtained from $10^{6}$ ${ }^{178 \mathrm{~m} 2} \mathrm{Hf}$ ion/s in a purified beam. It may then be possible, for example, to look for Coulomb excitation, through a $0.33 \mathrm{MeV}, \mathrm{E} 2$ transition, to the I $=14$ member of the ground-state band [24], and thereby test the high degree of $\mathrm{K}$ mixing that has been inferred by Hayes et al. [25]. This would be a form of isomer photo-destruction, but now with a substantial energy release $(2.45 \mathrm{MeV})$ compared to the energy of excitation $(0.33 \mathrm{MeV})$.

However, a different perspective should also be considered. Once isomer beams are under discussion, then ${ }^{178 \mathrm{~m} 2} \mathrm{Hf}$ is not so special from an experimental point of view. There are numerous highly excited isomers with $T_{1 / 2}>500 \mathrm{~ns}$, surviving long enough for mass separation and/or ion-by-ion A/q identification. A selection of such isomers, with energy $>2 \mathrm{MeV}$, is illustrated in Figure 4.

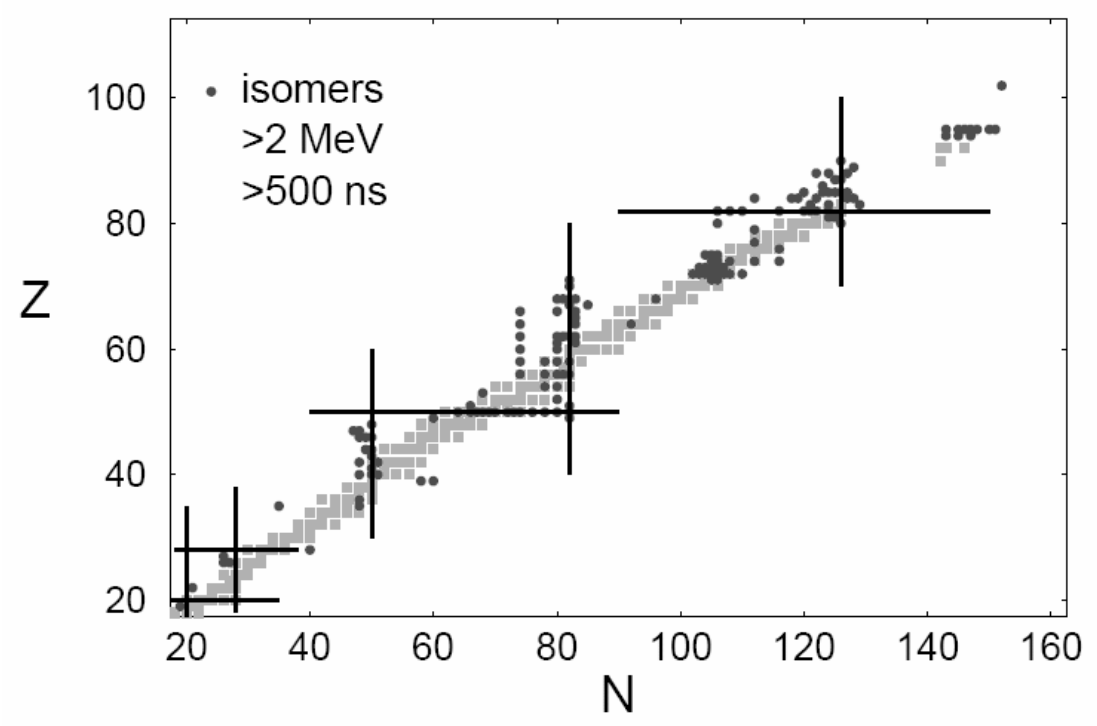

Figure 4: Chart of nuclides, illustrating a selection of highly excited isomers (dots) that are long enough lived for secondary-reaction measurements. Naturally occurring nuclides are shown as squares.

Pioneering experiments at RIKEN, Japan, have been performed with isomer beams, produced using inverse heavy-ion, fusion-evaporation reactions in conjunction with a recoil filter. Measurements include the Coulomb excitation of a $2 \mu \mathrm{s}, \mathrm{K}=8$ isomer in ${ }^{174} \mathrm{Hf}[26]$, and secondary fusion reactions with a $1 \mu \mathrm{s}, \mathrm{I}=49 / 2$ isomer in ${ }^{145} \mathrm{Sm}$ [27]. However, to date, the limitations of low beam intensity, low beam purity and poor beam optics have placed severe restrictions on the quality of the information that can be obtained. Now we are entering a new era for radioactive beams in general, and for isomer beams in particular. A key aspect for isomer beams will be the ability to separate the isomeric states from their respective ground states, and several methods are available. 
An outstanding capability has already been demonstrated at ISOLDE, CERN. Copper isomers and ground states have been separated by laser resonance ionisation. In the case of ${ }^{68} \mathrm{Cu}$, a purified beam of the $\mathrm{I}=6$ isomer $\left(\mathrm{T}_{1 / 2}=4 \mathrm{~m}, 722 \mathrm{keV}\right)$ was

accelerated to $200 \mathrm{MeV}$ and Coulomb excited by ${ }^{120} \mathrm{Sn}$ target nuclei [28]. A notable feature was the detection of a $\gamma$-ray cascade back to the ground state, bypassing the isomer. This ISOL method requires an isomer with $\mathrm{T}_{1 / 2} \geq 1 \mathrm{~s}$.

Also requiring second or longer half-lives is the storage-ring technique developed at GSI for fragmentation reaction products [29]. The different orbits of electron-cooled beams have been shown to give the capability of isomer separation by insertion of a mechanical "scraper". Alternatively, there are special cases such as ${ }^{212} \mathrm{Po}$, where the ground state is short lived $(0.3 \mu \mathrm{s})$ compared to the isomer $(45 \mathrm{~s})$ so that a ${ }^{212 \mathrm{~m}} \mathrm{Po}$ beam would already be purified by the time it was cooled. Finally, for $\mu$ s isomers it is possible to use delayed-coincidence techniques to select isomer-related events, for example by requiring Coulomb excitation $\gamma$ rays to be time correlated with $\gamma$ rays that come from the isomer decay. The many possibilities need careful evaluation.

\section{Atomic-nuclear interface}

The 7 hour, $\mathrm{I}=21 / 2$ isomer in ${ }^{93} \mathrm{Mo}$, at $2.43 \mathrm{MeV}$, presents an unusual situation, illustrated in Figure 5. The structurally related 17/2 state is just $5 \mathrm{keV}$ higher in energy than the isomer. It has been estimated that, in a plasma at a temperature above $5 \times 10^{6} \mathrm{~K}$, the isomer decay rate would be enhanced by a factor of $\sim 10^{6}$, largely due to the predicted NEEC (nuclear excitation by electron capture) process [30]. NEEC is the inverse of electron conversion, which is well known often to dominate over $\gamma$-ray emission for low-energy transitions, but NEEC itself has yet to be observed in any nuclide. The 7 hour isomer in ${ }^{93} \mathrm{Mo}$ seems to be a candidate for a radioactive beam with which to explore such a process. Indeed, the release of $2.43 \mathrm{MeV}$ isomer energy initiated by a $5 \mathrm{keV}$ excitation could have wider interest. This is just one example of using an isomer to give a prominent signal (several high-energy $\gamma$ rays) when excited by relatively low-energy photons. There are many more possibilities represented in Figure 4.

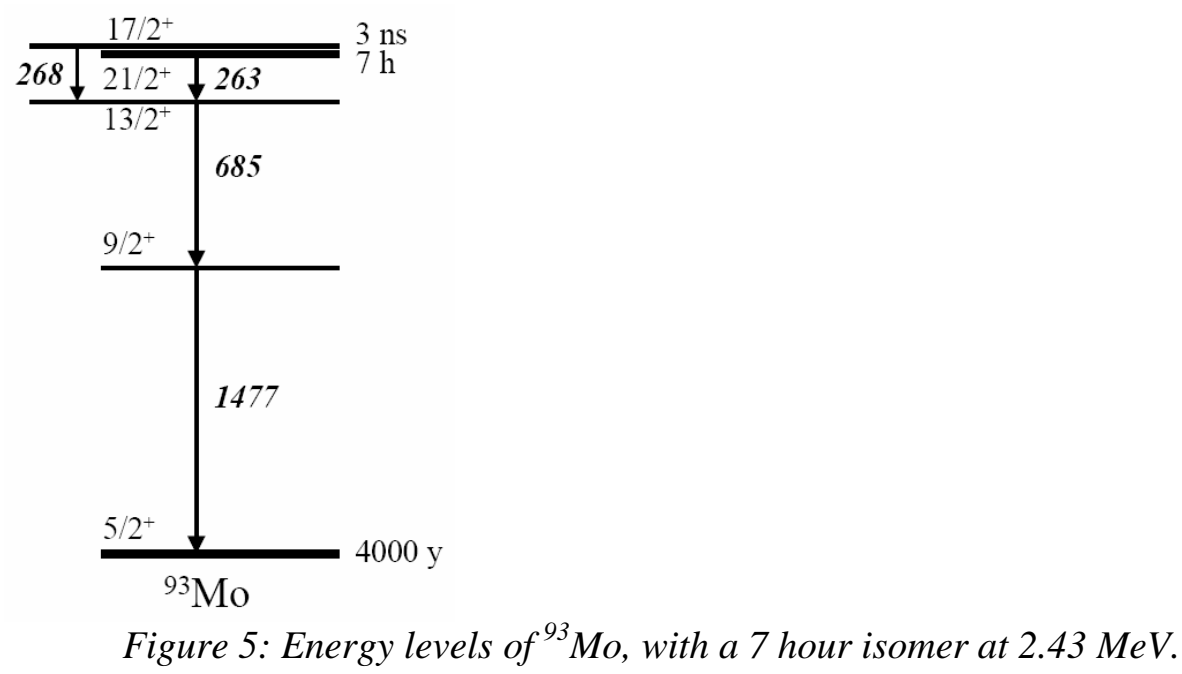




\section{Gamma-ray laser}

Isomers have been associated with the quest for a $\gamma$-ray laser, a long-standing dream that is perhaps as challenging as the search for controlled fusion power. The first concrete suggestions for a laser that utilized nuclear excited states as upper and lower lasing levels appeared in the literature in 1963 [31], following an earlier, previously classified Russian patent [32]. It is interesting how soon these works came after the first demonstration of optical lasing, but perhaps more amazing is that the fundamental recognition that stimulated emission was possible in nuclei came as early as 1926 [33].

A $\gamma$-ray laser would require a difficult synthesis of natural parameters and technical achievements, a challenge that has not yet been surmounted. In any laser, the most basic requirement is a population inversion between paired quantum states that serve as upper and lower laser levels. Stimulated emission of the corresponding electromagnetic decay transition gives the laser radiation, with repeated re-excitation of the upper laser level "pumping" the inversion and repeated stimulation of the emission increasing the amplification. Such a repetition is unlikely for a $\gamma$-ray laser, due to the difficulty in providing efficient reflection of high-energy photons.

It is in the preparation of a population inversion that isomers have been proposed to play a major part. The greatest impediment to the creation of a $\gamma$-ray laser has been the inherent conflict (the "graser dilemma" [34]) between the pumping of a population inversion and the requirement that the linewidth for the lasing transition be maintained as close to the natural width as possible. Various methods have been suggested by which to preserve the natural transition linewidth, such as using the Mössbauer effect or laser-cooled ensembles, but strong irradiation of a laser material to provide an inversion could easily destroy the narrow linewidth. A nuclear analogue of the standard four-level laser scheme, with nuclei beginning in a long-lived isomer instead of the ground state, might reduce the pump fluxes (by photons, neutrons or ions) to a level that preserves the linewidth. So far, no system of suitable levels with the necessary transition probabilities and lifetimes has been found in any nuclide. It is worth mentioning that an extension of atomic processes like quantum interference might be possible, eliminating the competing absorption of laser photons by the lower laser level in a process called gain without inversion [35].

The field of $\gamma$-ray lasers thus remains dependent on further innovation. One key step would be the demonstration of isomer energy release induced by low-energy $(<<1$ $\mathrm{MeV}$ ) photons [36]. This is where, as discussed earlier, radioactive beams may be needed.

\section{Outlook}

It is well recognised that radioactive beams are opening up the nuclear landscape. In this brief review, we have tried to show that isomers give an added dimension to these radioactive-beam developments, further extending the nuclear physics horizons.

Moreover, isomer beams will offer unique possibilities to explore new physics at the atomic-nuclear interface. We must expand our mental horizons, not just our physical horizons, if we are fully to exploit the opportunities, and there is plenty of scope for new ideas. 
This work has been supported by the UK EPSRC and AWE, and by the US AFOSR under contract FA9550-05-1-0486.

[1] L. Carroll, Through the Looking Glass (Macmillan and Co., London, 1872).

[2] F. Soddy, Nature 99, 433 (1917).

[3] A. Bohr and B. R. Mottelson, Phys. Rev 90, 717 (1953).

[4] P. M. Walker and G. D. Dracoulis, Nature 399, 35 (1999).

[5] P. M. Walker and G. D. Dracoulis, Hyperfine Interactions 135, 83 (2001).

[6] P. M. Walker and J. J. Carroll, Physics Today 58, 39 (June 2005).

[7] G. D. Dracoulis et al., Phys. Rev. Lett. 97, 122501 (2006).

[8] S. Shimoura et al., Phys. Lett. B560, 31 (2003).

[9] I. Mukha et al., Phys. Rev. Lett. 95, 022501 (2005); Nature 439, 298 (2006).

[10] A. Kuhnert et al., Phys. Rev. C46, 484 (1992).

[11] M. Hult et al., Phys. Rev. C74, 054311 (2006).

[12] B. R. Beck et al., Phys. Rev. Lett. 98, 142501 (2007).

[13] S. Hofmann et al., Eur. Phys. J. A10, 5 (2001).

[14] V. V. Flambaum, Phys. Rev. Lett. 97, 092502 (2006).

[15] F. R. Xu et al., Phys. Rev. Lett. 92, 252501 (2004).

[16] R.-D. Herzberg et al., Nature 442, 896 (2006).

[17] P. H. Regan et al., Nucl. Phys. A787, 491c (2007).

[18] D. Belic et al., Phys. Rev. Lett. 83, 5242 (1999).

[19] P. M. Walker, G. D. Dracoulis and J. J. Carroll, Phys. Rev. C64, 061302R (2001).

[20] P. Mohr, F. Käppeler and R. Gallino, Phys. Rev. C75, 012802R (2007).

[21] S. Deylitz et al., Phys. Rev. C53, 1266 (1993).

[22] E. V. Tkalya, Phys. Rev. C71, 024606 (2005).

[23] I. Ahmad et al., Phys. Rev. C71, 024311 (2005).

[24] S. A. Karamian and J. J. Carroll, Laser Phys. 17, 80 (2007).

[25] A. B. Hayes et al., Phys. Rev. Lett. 96, 042505 (2006).

[26] T. Morikawa et al., Phys. Lett. B350, 169 (1995).

[27] H. Watanabe et al., Nucl. Phys. A746, 540c (2004).

[28] G. Georgiev et al., Int. J. Mod. Phys. E15, 1505 (2006); I. Stefanescu et al., Phys. Rev. Lett. 98, 122701 (2007).

[29] F. Bosch et al., Int. J. Mass Spec. 251, 212 (2006).

[30] G. Gosselin and P. Morel, Phys. Rev. C70, 064603 (2004).

[31] G. C. Baldwin, J. P. Neissel and L. Tonks, Proc. IEEE 51, 1247 (1963); V. Vali and W. Vali, Proc. IEEE 51, 182 (1963).

[32] L. A. Rivlin, Soviet Inventor's Certificate No. 621265, 10 January 1961.

[33] A. S. Eddington, The Internal Constitution of the Stars (Cambridge Univ. Press, London, 1926).

[34] G. C. Baldwin and J. C. Solem, Rev. Mod. Phys. 69, 1085 (1997).

[35] O. Kocharovskaya, R. Kolesov and Y. Rostovtsev, Laser Phys. 9, 745 (1999).

[36] J. J. Carroll et al., Hyperfine Interactions 135, 3 (2001). 


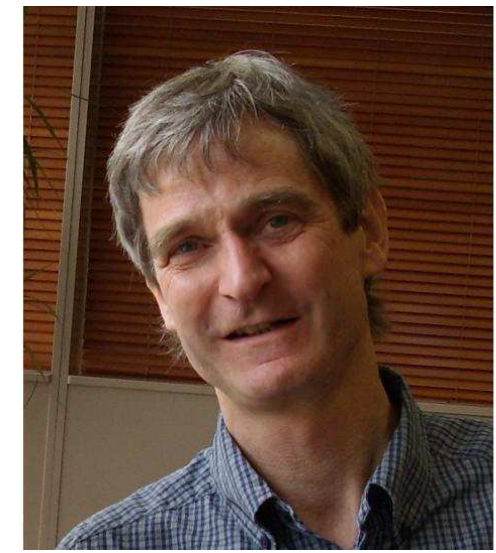

Phil Walker

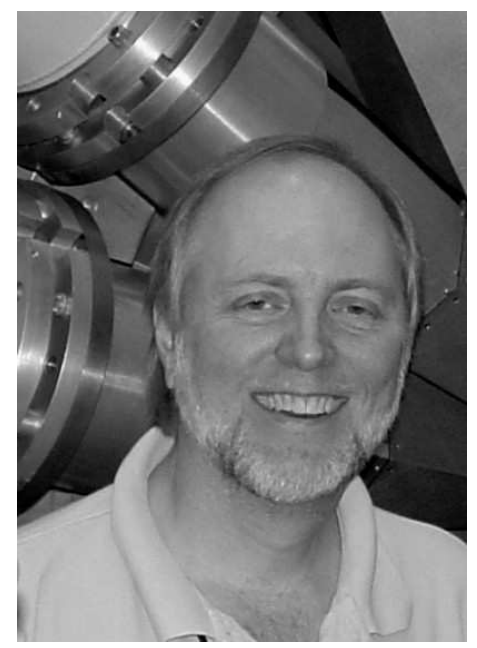

James Carroll

Corresponding author: Phil Walker: phone +44-1483-686807; fax +44-1483-686781 Email: p.walker@surrey.ac.uk

This is a preprint. The final version is published in Nuclear Physics News 17, No.2, 11-15 (2007). 